\title{
Elastic Relaxation of Strained Silicon on Insulator (sSOI) Fins: Nanobeam Diffraction (NBD) and Simulations
}

\author{
J. Li ${ }^{1}$, Pierre Morin ${ }^{2}$, Q. Liu ${ }^{2}$, K. Cheng ${ }^{1}$, N. Loubet ${ }^{2}$, B. Doris ${ }^{1}$, J. Gaudiello ${ }^{1}$ \\ ${ }^{1}$ IBM, Albany, NY 12203 USA \\ ${ }^{2}$ STMicroelectronics, Albany, NY 12203 USA \\ juntaoli@us.ibm.com
}

Channel strain engineering has been used since the 90nm node [1]. However, as technology scales, the effectiveness of conventional strain elements (such as Source/Drain stressors, stress liners) is significantly reduced. Intrinsically strained channel materials such as tensile strained-silicon on insulator (sSOI) and compressive strained SiGe, have been applied on planar devices [2, 3] to boost transistors. However, it is critical to understand the impact of Fin geometry on elastic relaxation in advanced 3D FinFETs structures. In this paper, we investigate the elastic relaxation in state-of-the-art sSOI Fins through Nano-Beam Diffraction (NBD) and calibrated 3D simulations.

Intrinsically strained $\mathrm{Si}$ on insulator was used: initial lattice matched to $\mathrm{Si}_{0.7} \mathrm{Ge}_{0.3}$. This sSOI is initially biaxially strained. After Fin patterning (i.e., etch and cut), we studied the evolution of the strain in the Fin structures. The reported results only account for elastic relaxation. Fig. 1 shows the cross scetional TEM views of the final Si Fins. Mechanical simulations were performed on Fin structures using a finite element method to solve the balance force equations. We used the nano-beam electron diffraction technique to determine the lattice deformation along the strained Si Fins formed on insulator (Fig. 2). Longitudinal strain along the length of a $380 \mathrm{~nm}$ Fin, at mid $\mathrm{H}_{\text {Fin, }}$, was then inferred from the $\mathrm{Si}$ substrate. The resulting profiles were used to validate mechanical simulation. Both measured and simulated profiles are shown on Fig. 2. An excellent matching is obtained.

Stress contour map of the half strain Si Fin was shown in Fig.3 (left) and the elastic relaxation is clearly visible at the edges of the Fin and is more pronounced at the top of the Fin. Little relaxation is seen at the Fin/BOX interface where the Fin material is anchored to the oxide. Fig. 3 (right) illustrates the vertical transversal stress gradients from Fin bottom to top. When the Fin is cut, the strain is not maintained along the edges. In order to evaluate the stress relaxations from sSOI Si Fins with various Fin lengths, Focused Ion Beam (FIB) was used to simulate the Fin Cut process to form Fins. LFin was varied between 100 and 1000nm. To be consistent with current Fin formation techniques, $\mathrm{L}_{\text {Fin }}$ was defined by a FIB cut ending on the BOX for SOI. Foused Ga ion beam with beam energy of $30 \mathrm{keV}$ and beam current of 50pA was used to cut inisde the macro with extreme long $(600 \mu \mathrm{m})$ parallal Fins. Fig.4a shows the low mag STEM view of the sample prepared using the method decribed above. Fig.4b shows a zoom in view of the 860nm Fin. Fig.5 (top) shows the in plane lattice deformation map taken from the $860 \mathrm{~nm}$ Fin (The refernce was taken from the center of sSOI Fin). Fig.5 (bottom) illustrates the in plane lattice deformation profile along long sSOI Fin, at mid $\mathrm{H}_{\mathrm{Fin}}$. The investigation of strain relaxation/distribution in shorter Fins is still ongoing. In addition, similar stress relaxation studies on SGOI (SiGe on Insulator) and bulk SiGe FinFET structures will also be discussed. More detailed NBD strain analysis and FEM mechanical simulations will be included in this work.

The NBD strain measurements correlated with FEM simulation on sSOI has been demonstrated that it can provide the high spatial resolution and high strain sensitivity for strain analysis in semiconductor 
materials. This technique can be used to investigate the elastic relaxation in state of the art sSOI Fins, SGOI and bulk SiGe FinFET devices.

\section{Reference}

[1]T. Ghani et al., IEDM, p.1161, 2003.

[2]A. Khakifirooz et al., VLSI, p.117, 2012.

[3] K. Cheng et al., IEDM, p.419, 2012.

Acknowledgement: This work was performed by the Research Alliance teams at various IBM Research and Development Facilities.

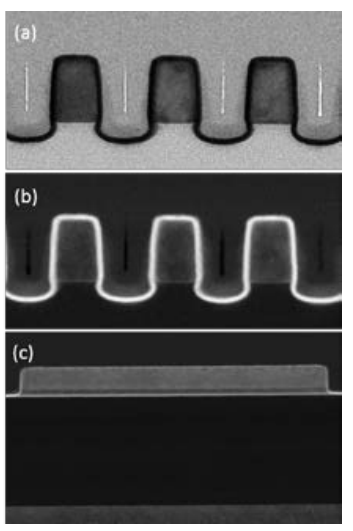

Fig.1 (a) TEM cross-sections of SSOI Fins; (b) dark field STEM image and (c) $380 \mathrm{~nm}$ long sSOI Fin.

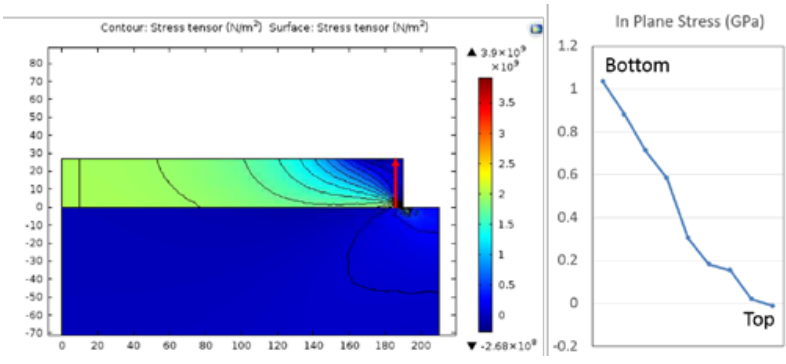

Fig.3 Stress contour map (left) and the plane stress profile from Fin bottom to top (right).

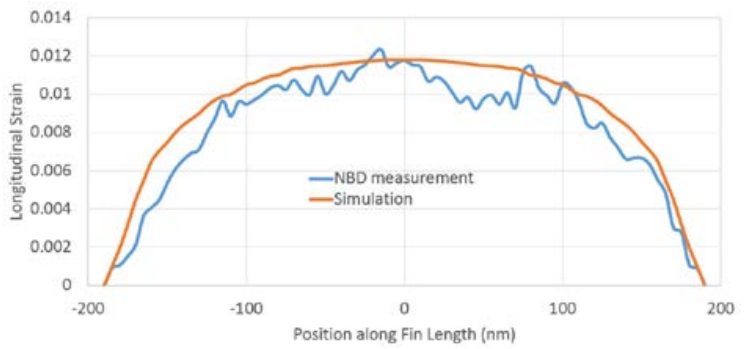

Fig.2 NBD measurement (blue line) and Simulation (orange line) of the longitudinal strain along the Fin length for a 380nm long sSOI Fin.
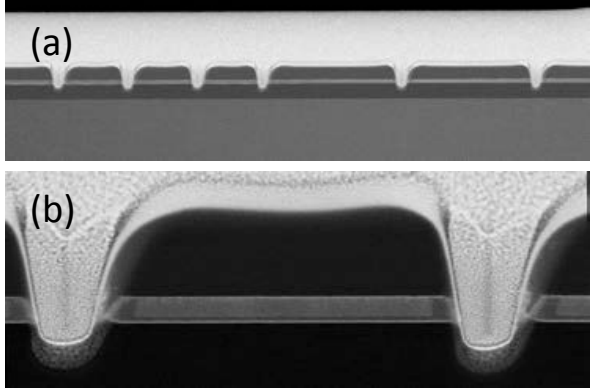

Fig.4 Simulated Fin Cut by Focused Ion Beam (FIB).

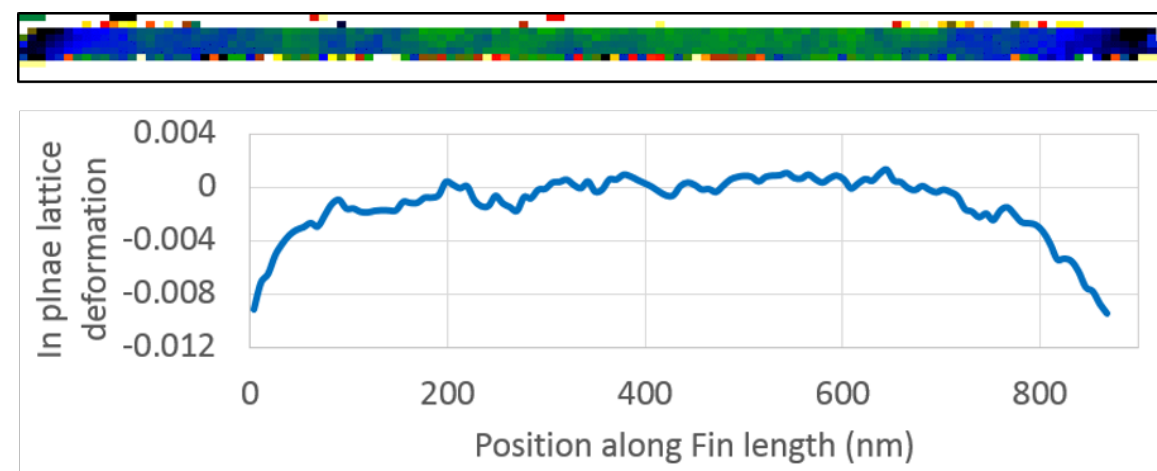

Fig.5 In plane lattice deforamtion map. The reference was taken from the center of sSOI Fin (top). In plane lattice deformation profile along long sSOI Fin at mid $\mathrm{H}_{\text {Fin }}$ (bottom) 